\title{
高-过成熟海相页岩中矿物-有机质复合体(MOA)的 显微激光拉曼光谱特征作为成熟度指标的意义
}

肖贤明 ${ }^{1,2^{*}}$, 周秦 ${ }^{2}$, 程鹏 ${ }^{2}$, 孙键 ${ }^{2}$, 刘德汉 ${ }^{2}$, 田辉 ${ }^{2}$

1. 中国地质大学(北京)能源学院, 北京 100083 ;

2. 中国科学院广州地球化学研究所有机地球化学国家重点实验室, 广州 510640

* 通讯作者, E-mail: xmxiao@cugb.edu.cn

收稿日期：2020-01-29; 收修改稿日期：2020-04-16; 接受日期：2020-05-08; 网络版发表日期：2020-07-21

国家自然科学基金项目(批准号：U19B6003、U1810201)、国家科技重大专项项目(编号：2017ZX05008-002-004)和中国科学院战略性先导科 技专项A类项目(编号: XDA14010104)资助

摘要 煤和沉积岩中有机质的显微激光拉曼光谱参数越来越多地应用于成熟度评价. 虽然针对纯固体有机质(如 镜质组、固体励青)及分离提取的干酪根作了大量的研究，但缺乏直接基于页岩全岩样品的矿物-有机质复合体 $(\mathrm{MOA})$ 的系统报道。文章通过对取自中国南方不同有机质含量 $(\mathrm{TOC}=0.10 \sim 4.59 \%)$ 与不同成熟度 $\left(B R_{\mathrm{o}}\right.$ $=1.71 \sim 4.57 \%)$ 的两套海相贡岩样品中MOA激光拉曼光谱的研究，发现该项技术对MOA 中赋在的有机质非常敏 感, 当页岩TOC含量达到 $0.60 \%$, 并可获得完美的有机质的拉曼图谱, 与其共生固体沥青的拉曼参数高度吻合, 同 样可指示页岩的成熟度水平. MOA的拉曼参数是评价高-过成熟页岩成熟度水平的潜在方法, 其最大优点是规避 了对页岩中显微组分的鉴定与篎选，特别适用于那些缺乏可用于反射率或拉曼测定显微组分的下古生界及前寒 武系海相页岩的成熟度评价.

关键词海相页岩, 矿物- 有机质复合体(MOA), 拉曼光谱参数, 固体沥青, 成熟度

\section{1 前言}

富有机质页岩中的固体有机质(统称为干酪根)在 普通光学显微镜下有两种存在形式: 一种是可分辨的 显微组分, 如镜质组、藻类组、壳质组、固体沥青、 惰性组、笔石及其碎屑(Teichmüller，1986; SuárezRuiz等，2012); 另一种是非常细小难鉴别的有机质 $(<1 \mu \mathrm{m})$ ，其分散于微细矿物(如黏土矿物、石英等)颗 粒间，构成矿物-有机质复合体(mineral-organic aggre- gation, MOA)(Chalmers等, 2012), 也即有机岩石学家 称之为的矿物沥青基质(Teichmüller, 1986). 这部分有 机质在制备的干酪根样品中基本呈无定形体，在 I 型 与 II 型干酪根为主的页岩中, 其含量一般占主导地位 (Xiao等, 1998).

成熟度是烃源岩评价的重要指标, 也是页岩油气 勘探开发的重要依据(Curtis，2002；Jarvie等，2007; Jarvie, 2012; Xiao等, 2015). 对于下古生界及前寒武系 海相页岩, 由于镜质组反射率 $\left(V R_{\mathrm{o}}, \%\right)$ 不能应用, 通常 
采用的成熟度指标是固体沥青反射率 $\left(B R_{0}, \%\right)$ 与笔石 体反射率 $\left(G R_{0}, \%\right)$ (Bertrand和Malo, 2001; Valentine等, 2014; Sanei等, 2015; Luo等, 2017). 然而, 并非所有页 岩中都可找到可供反射率测定的固体沥青与笔石体 (Petersen等, 2013), 也不是这两种显微组分的反射率均 适合作为成熟度指标，其明显受来源、颗粒大小及光 学各向异性的制约(Schoenherr等，2007; Suárez-Ruiz 等, 2012; Sanei等, 2015; Luo等, 2019). 由于缺乏有效 的成熟度指标，下古生界及前寒武系海相页岩的成熟 度评价没有得以有效的解决, 并一直在研究与探索 (Caricchi等, 2016; Luo等, 2019).

激光拉曼光谱被广泛应用于表征地质含碳物质的 结构有序度(Cuesta等, 1994; Bustin等, 1995; Ferrari和 Robertson, 2000; Beyssac等, 2002, 2003; Sadezky等, 2005; Bernard等, 2010), 并主要应用于指示其母岩所经 历的地质温度(Beyssac等, 2002; Rahl等, 2005; Aoya等, 2010；Endo等，2012)或者成熟度(Kelemen和Fang, 2001; Jehlicka等，2003; Quirico等，2005; Guedes等, 2010; 刘德汉等, 2013; Hinrichs等, 2014; Wilkins等, 2014；Zhou等，2014; Lünsdorf，2016; Mumm和Inan, 2016). 研究对象主要包括碳质变质岩(Beyssac等, 2002; Aoya等，2010)、碳质球粒陨石(Ellery等，2004; Popp和Schmitt，2004)、煤(Kelemen和Fang，2001; Quirico等，2005; Marques等，2009; Guedes等，2010; Sonibare等, 2010)、固体沥青(Jehlicka等, 2003; Court 等, 2007; 刘德汉等, 2013; Zhou等, 2014)及含碳化石 (Schopf等, 2005; Schopf和Kudryavtsev, 2009; Engdahl 等, 2015; Ferralis等, 2016; Mumm和Inan, 2016; Luo等, 2017). 近年来，激光拉曼技术拓展到对含油气盆地页 岩中显微组分及全岩干酪根的研究(Kelemen和Fang, 2001; Zeng和Wu, 2007; Wilkins等, 2014, 2015; Zhou等, 2014; Lünsdorf, 2016; Schito等, 2017; Henry等, 2018, 2019a)，并应用于指示页岩成熟度也取得了一定的进 展. 下面是一些研究实例. Wilkins等 $(2014,2015)$ 提出 了一种基于激光拉曼参数的成熟度表征方法(称之为 RaMM方法). 该方法应用基于几项拉曼参数的两个回 归方程来计算等效镜质组反射率(适用于 $V R_{\mathrm{o}}$ 范围: 0.4 2.5\%), 并证明该方法可以有效解决海相页岩中广 泛存在的 $V R_{\mathrm{o}}$ 抑制问题. Schito等(2017)开展了低-中成 熟度 $\left(V R_{0}\right.$ 范围: $\left.0.35 \sim 1.50 \%\right)$ 全岩干酪根样品的激光拉 曼研究, 证明了一系列拉曼光谱参数与埋藏深度(或
$V R_{\mathrm{o}}$ )均具有很好的相关性. 还有一些研究(如Zhou等, 2014; Sauerer等, 2017; Henry等, 2019b)致力于激光拉 曼成熟度参数的篮选, 发现在众多潜在的参数中, RBS ( $\mathrm{G}$ 峰位与 $\mathrm{D}$ 峰位的差值)在 $V R_{\mathrm{o}}$ 约为 $0.50 \sim 3.50 \%$ 的范围 内是评价页岩成熟度最好的参数. 普遍认为激光拉曼 参数有可能成为继镜质组反射率之后另一项可广泛应 用于煤和页岩中有机质的成熟度快速且无损伤测定技 术(Hinrichs等，2014; Zhou等，2014; Lünsdorf, 2016; Sauerer等，2017; Schito等，2017; Henry等，2018， 2019b).

镜质组是一种广泛接受可应用于沉积岩中激光拉 曼成熟度测定的显微组分(Lünsdorf，2016；Henry等， 2019a). 然而，镜质组在一些优质生油岩中相对罕见， 在下古生界及前寒武系页岩中缺乏(Teichmüller, 1986). 在这些情况下, 需要采用干酪根样品应用于激 光拉曼成熟度测定(Schito等, 2017). 众所周知, 从页岩 中获取干酪根的过程不仅时间长、成本高，而且还可 能受样品数量的限制(为获得足够数量的干酪根, 通常 需要50 100g页岩样品), 有时难以实施, 因此, 以干酪 根为基础的激光拉曼成熟度参数很难广泛推广应用.

Schopf等(2005)研究了前寒武系岩石中矿化化石 的激光拉曼光谱特征. 他们通过对这些嵌入矿物中含 碳化石的原位分析获得了完美反映有机质的拉曼吸附 峰, 并发现矿物介质的影响非常有限. 该项研究为采用 页岩光片直接测定矿物中包裹的干酪根亚显微组分的 激光拉曼光谱提供了启示. 本研究直接测定了不同 TOC(总有机碳)含量和不同成熟度的海相页岩样品中 MOA的激光拉曼光谱, 研究了MOA中矿物背景荧光 和有机质的非均质性对其激光拉曼光谱特性与参数的 影响, 并通过与固体沥青的比较, 探讨了 $\mathrm{MOA}$ 的激光 拉曼参数作为成熟度指标的意义.

\section{2 样品与实验}

\section{1 样品}

研究样品除了M1外, 均取自中国南方下古生界广 泛分布的两套海相页岩: 下寒武统筇竹寺组与上奥陶 统五峰组-下志留统龙马溪组(邹才能等, 2010). M1样 品取自四川盆地GK1井的下三叠统海相页岩, 主要目 的是为增加所研究的成熟度范围(表1). 研究样品包括 两个系列：一个是取自 PY1井与 QJ井的 TOC系列 
肖贤明等: 高-过成熟海相页岩中矿物-有机质复合体(MOA)的显微激光拉曼光谱特征作为成熟度指标的意义

表 1 样品基础地质与地球化学信息 ${ }^{a)}$

\begin{tabular}{|c|c|c|c|c|c|c|}
\hline 样品编号 & 井号或剖面 & 埋藏深度 $(\mathrm{m})$ & 岩石名称 & 地层年代 & TOC $(\%)$ & $B R_{\mathrm{o}}(\%)$ \\
\hline $\mathrm{T} 1$ & 贵州PY-1井 & 2126 & 黑色页岩 & 下志留统 & 1.01 & I \\
\hline $\mathrm{T} 2$ & 贵州PY-1井 & 2130 & 黑色页岩 & 下志留统 & 1.56 & l \\
\hline $\mathrm{T} 3$ & 贵州PY-1井 & 2136 & 黑色页岩 & 下志留统 & 2.62 & 3.49 \\
\hline $\mathrm{T} 4$ & 贵州PY-1井 & 2140 & 黑色页岩 & 下志留统 & 3.47 & 1 \\
\hline $\mathrm{T} 5$ & 贵州PY-1井 & 2149 & 黑色页岩 & 下志留统 & 3.98 & 3.56 \\
\hline $\mathrm{T} 6$ & 贵州PY-1井 & 2155 & 黑色页岩 & 下志留统 & 4.59 & 3.51 \\
\hline $\mathrm{T} 7$ & 贵州PY-1井 & 2160 & 灰色泥岩 & 下志留统 & 0.10 & / \\
\hline $\mathrm{T} 8$ & 重庆黔江1井 & 718 & 灰黑色泥岩 & 下志留统 & 0.39 & 3.41 \\
\hline T9 & 重庆黔江1井 & 772 & 灰黑色泥岩 & 下志留统 & 0.60 & 3.49 \\
\hline M1 & 四川高科1井 & 3146 & 灰黑色泥岩 & 下三叠统 & 4.67 & 1.71 \\
\hline M2 & 四川广安华菳灵峰剖面 & l & 黑色泥岩 & 上奥陶统 & 9.12 & 2.33 \\
\hline M3 & 贵州遵义松林剖面 & l & 黑色泥岩 & 下寒武统 & 4.35 & 2.58 \\
\hline M4 & 南京幕府山剖面 & l & 黑色泥岩 & 下寒武统 & 3.44 & 2.93 \\
\hline M5 & 贵州习水县骑龙村剖面 & 1 & 黑色泥岩 & 上奥陶统 & 6.06 & 3.16 \\
\hline M6 & 贵州黄页1井 & 2421 & 黑色泥岩 & 下寒武统 & 13.7 & 3.50 \\
\hline M7 & 贵州彭页1井 & 2144 & 黑色页岩 & 下志留统 & 3.41 & 3.58 \\
\hline M8 & 四川高科1井 & 4986 & 黑色页岩 & 下寒武统 & 1.62 & 3.73 \\
\hline M9 & 重庆南川大有镇剖面 & / & 黑色泥岩 & 下志留统 & 1.08 & 3.88 \\
\hline M10 & 贵州凤参1井 & 2144 & 黑色岩 & 下寒武统 & 2.54 & 3.95 \\
\hline M11 & 安徽泾县唐村剖面 & l & 灰黑色岩 & 下寒武统 & 2.92 & 4.35 \\
\hline M12 & 安徽巢湖剖面 & / & 黑色页岩 & 下寒武统 & 14.54 & 4.57 \\
\hline
\end{tabular}

a) /, 没有数据

(T1 T9), 这些样品成熟度相近， $B R_{\mathrm{o}}$ 介于 $3.41 \sim 3.56 \%$, 但TOC不同，TOC介于 $0.10 \sim 4.59 \%$ (表1; 图1); 另一个 为成熟度系列 (M1 M 12), 这些样品 TOC介于 $1.08 \sim 14.54 \%, B R_{0}$ 介于1.71 4.57\%, 分别取自 11 个不同 的地点(表1; 图1).

\section{2 实验}

按照有机岩石学研究的要求, 将页岩样品制备成 抛光的光片. 采用HORIBA-JY LabRAM全自动显微激 光拉曼光谱仪对样品中的MOA与固体沥青进行激光 拉曼光谱测定. 设置的主要实验条件为: 激光波长 $532 \mathrm{~nm}$ ，曝光时间 $10 \sim 20 \mathrm{~s}$, 样品表面激光强度 $0.45 \mathrm{~mW}$, 观测物镜 $\times 50$, 测点直径 $2 \mu \mathrm{m}$, 扫描波数范围 500 3000 $\mathrm{cm}^{-1}$ (详见刘德汉等, 2013; Zhou等, 2014; 王 茂林等, 2015). 采用硅片标样进行测定波数校准. 为减 少样品非均质性的影响, 选择的MOA测点不包含任何
可鉴别的显微组分或动物壳屑体的碎屑. 每个样品测 定 5 个不同的固体沥青颗粒与MOA，相关参数取其平 均值.

\section{3 曲线拟合}

纵观文献报道, 含碳物质激光拉曼光谱曲线的拟 合有一个值得思考的发展历程. 从早期的两个基本峰 (D峰与 G峰)的拟合(Green等, 1983; Bustin等, 1995; Kelemen和Fang, 2001), 发展到多峰拟合, 如3峰(Sonibare等，2010)、4峰(Aoya等，2010)、5峰(Sauerer等, 2017)、6峰及更多(Li等，2006; Ferralis等，2016; Guedes等, 2010; Schito等, 2017), 其目的用以获得拟 合峰之和与原图谱具有最大的拟合程度. 近年来, 从成 熟度的表征与实际应用考虑, 简单的两峰拟合方法又 在倡导与应用(Hinrichs等, 2014; Liu等, 2014; Wilkins 等, 2014, 2015; 王民和Li, 2015; Schmidt等, 2017), 甚至 


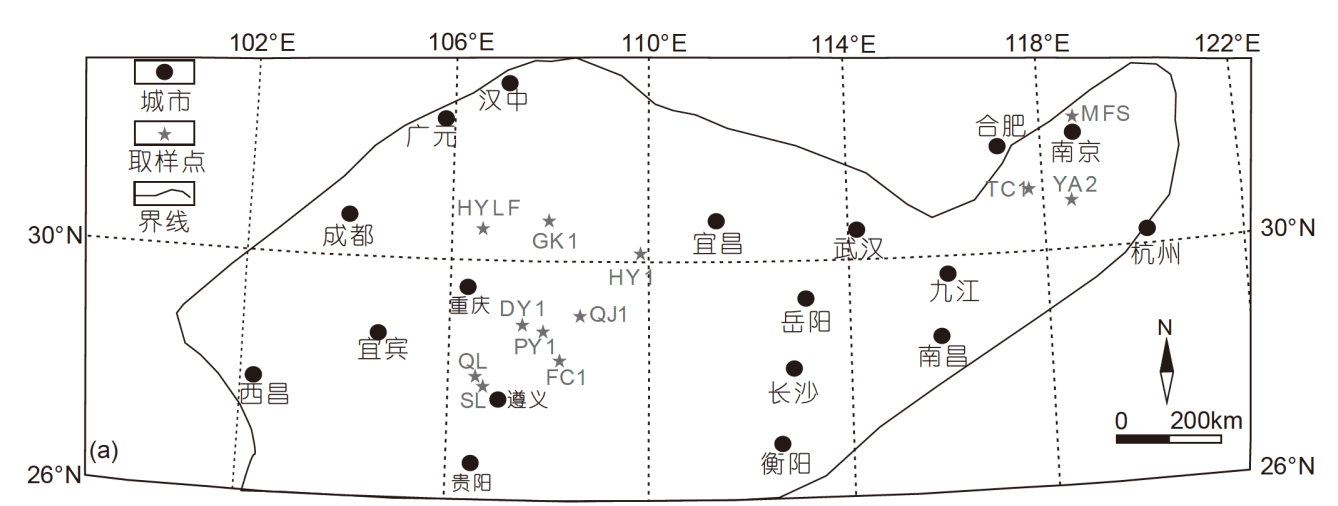

(b)

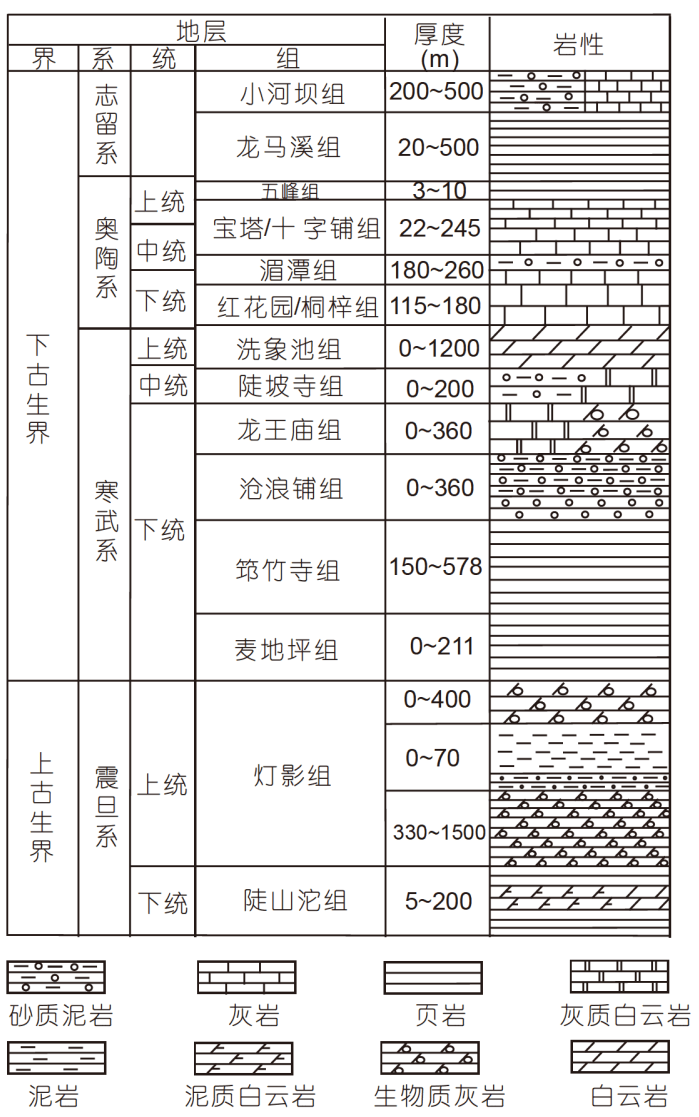

图 1 取样位置示意图(a)和中国南方下古生界地层柱状图(b)

(b)根据邹才能等(2010)简化

认为最好不要拟合, 这样可排除拟合过程中加工偏差 的影响, 获得更为稳定的参数, 同时可使得这项原本 简单的技术具有更好的可操作性与实用性(Henry等, 2018).

本研究采用仪器自带软件的GaussLoren模型, 采 用直线基线，对 $\mathrm{D}$ 峰与 $\mathrm{G}$ 峰进行直接拟合. 没有对 $\mathrm{D}$ 峰 与 $\mathrm{G}$ 峰作进一步分解, 不仅仅是因为分解过程具有很
大的不确定性(Quirico等, 2005, Court等, 2007; Kouketsu等, 2014; Lupoi等, 2017; Henry等, 2018), 而且本研 究的目的是评估MOA的激光拉曼光谱参数是否类似 于纯有机质(如固体沥青)同样适合作为成熟度指标, 同时便于与基于两峰拟合的已发表数据进行直接对比 (刘德汉等, 2013; Zhou等, 2014; 王茂林等, 2015). 此外, MOA的激光拉曼光谱实际上反映的是不同亚显微组 
分的混合物. 当采用多峰拟合方法解析单一显微组分 的激光拉曼光谱时，有助于将这些解析峰与该显微组 分的结构特征相关联. 但是，从混合显微组分获得的 激光拉曼光谱的结构解析太复杂，采用多峰拟合没有 实际意义.

图2为选择的三个不同成熟度样品MOA图谱的拟 合结果 $\left(R^{2}\right.$ 为 $\left.0.98 \sim 0.99\right)$, 拟合曲线基本上保持了原始 图谱形态，拟合效果类似于Henry等(2018)所建议的方 法M1(其要点是: G峰与D峰不拟合, 仅作平滑处理). 本 研究计算的激光拉曼光谱参数包括: 峰位 $\left(W_{\mathrm{D}}\right.$ 与 $\left.W_{\mathrm{G}}\right)$ 、 两峰间距 $\left(\mathrm{RBS}=W_{\mathrm{G}}-W_{\mathrm{D}}\right)$ 、半高宽 (FWHM-G 和 FWHM-D)、峰强度比 $\left(I_{\mathrm{D}} / I_{\mathrm{G}}\right)$ (I 为峰高, 后面相同). 对比 分析表明, 本研究拟合方法与M1方法所得的峰位与峰 强度基本上没有差别, FWHM-D与FWHM-G的差别分 别在 $\pm 5 \%$ 与 $\pm 1 \%$ 以内.

\section{3 结果与讨论}

\subsection{TOC 含量对MOA的激光拉曼光谱的影响}

根据干酪根显微组分组成与碳同位素数据，中国 南方下古生界海相页岩有机质的原始母质干酪根类型 主要为 I 型与 II a型(董大忠等, 2014; 刘树根等, 2016),

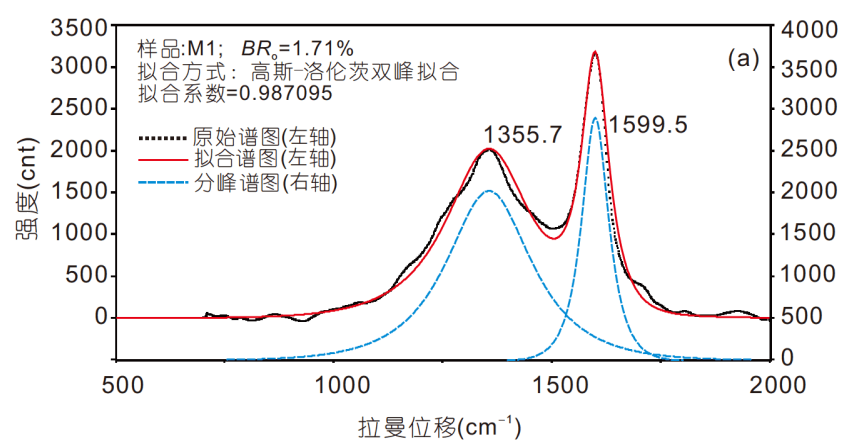

页岩中的干酪根主要以MOA的形式赋存, 其含量一般 可超过80 90\%(杨斌等，1996; Xiao等，2007; 张林等， 2008). 图3为不同TOC含量页岩样品的显微照片. 可见 可鉴别的显微组分主要是固体沥青、笔石及类镜质 组. 页岩的TOC含量越高, 固体沥青及其他可识别的显 微组分的数量越多(如M6样品, 图3f). 对于TOC含量较 低的页岩样品 $(<1.0 \%)$, 固体沥青含量很少. 因此, 对于 本研究TOC含量较高的样品, MOA中平均TOC含量要 明显低于样品的TOC含量. 此外, 由于页岩显微结构的 非均质性, 干酪根在矿物中的分布也不均一. 尽管如 此, 对于本研究样品, 其全岩TOC含量也可间接反映 出其MOA中的TOC含量的相对高低.

直接测定矿物包裹有机质的激光拉曼光谱时会 发现矿物的影响主要表现为较高的苂光背景, 同时在 $\mathrm{D}$ 峰上形成一些凸起(Schopf等, 2005). 页岩中的MOA 同样含有矿物和有机质，因此应首先讨论TOC含量对 MOA激光拉曼光谱的影响. T1 T9样品的成熟度相 似，但TOC含量差异很大(0.10 4.59\%)(表1), 可对其 激光拉曼光谱特征和参数进行比较与分析. 图 4 为 4 个 低 TOC含量样品的激光拉曼谱图. 虽然样品 T7 $(\mathrm{TOC}=0.10 \%)$ 具有清楚的 $\mathrm{D}$ 峰和 $\mathrm{G}$ 峰，但石英或碳酸 盐矿物的背景荧光导致拉曼谱图的基线随拉曼位移

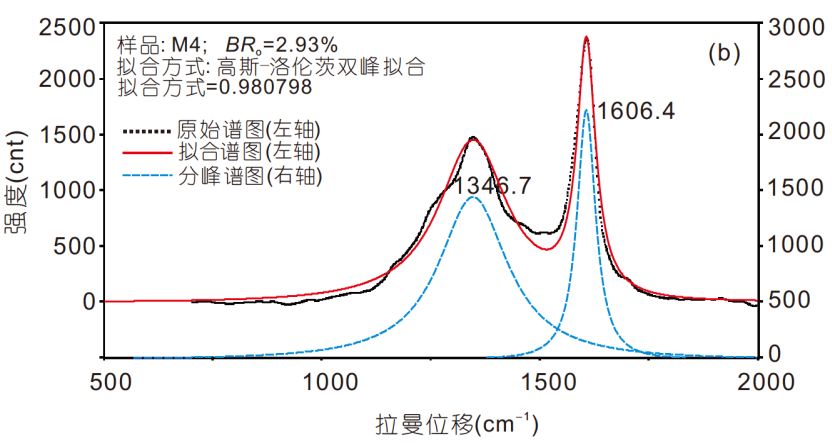

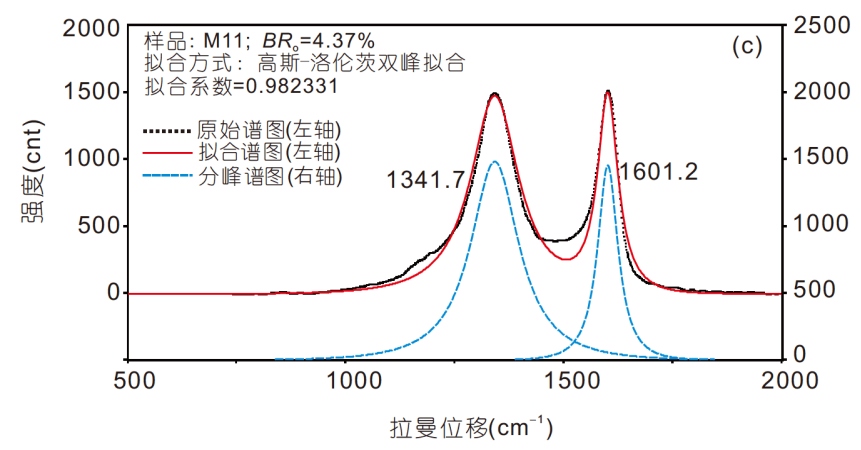

图 2 三个不同成熟度页岩样品的MOA图谱及其拟合效果 

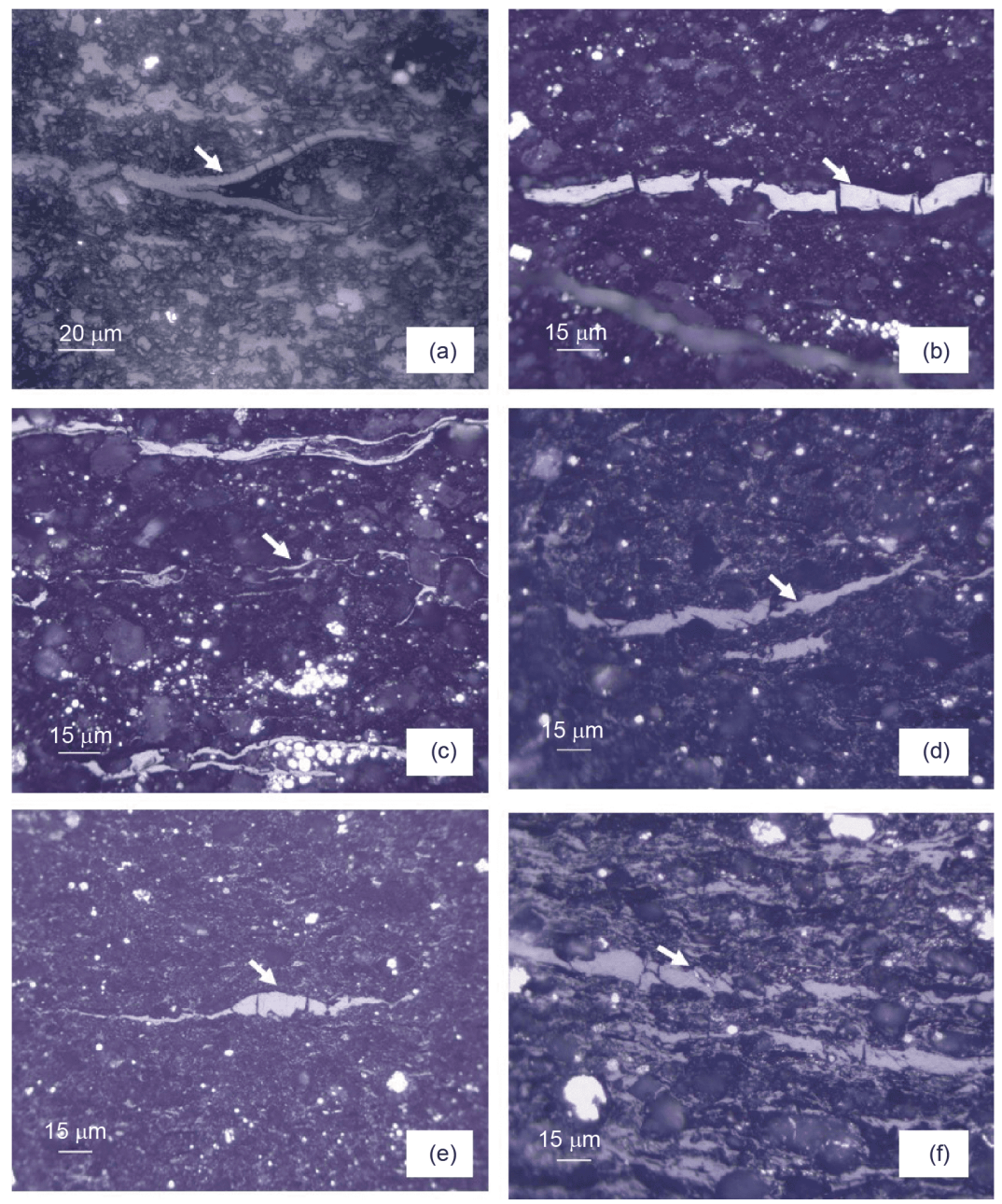

图 3 不同 TOC含量页岩样品的显微照片

(a) 笔石体, T7, TOC $=0.11 \%$; (b) 笔石体, T3, TOC $=2.62 \%$; (c) 固体沥青, T6, TOC $=4.59 \%$; (d) 固体沥青, M4, TOC $=3.44 \%$; (e) 固体沥青, M9, TOC $=3.88 \%$; (f) 固体沥青, M6, TOC $=13.70 \%$

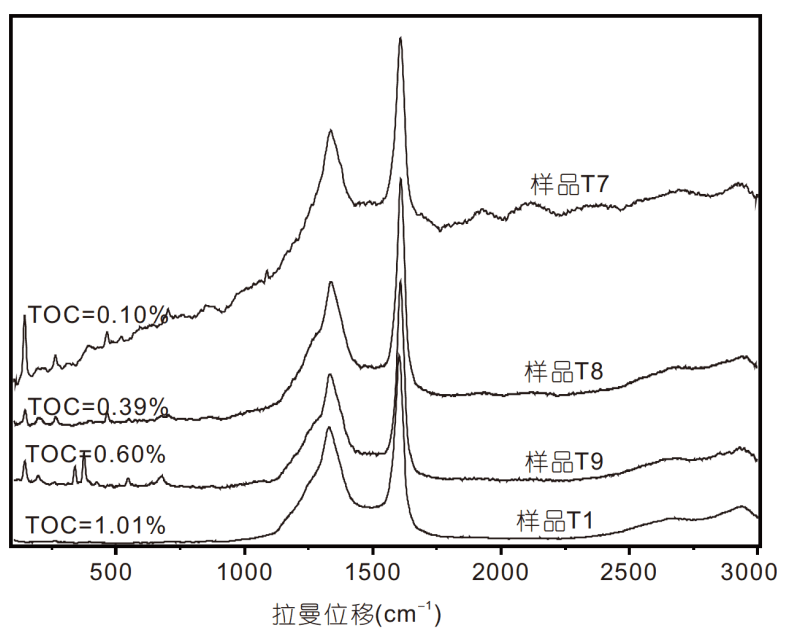

图 4 低TOC含量页岩样品的MOA激光拉曼谱图
的增加显著抬升, 同时在拉曼位移 $<500 \mathrm{~cm}^{-1}$ 出现矿物 的尖峰, 表明矿物的影响明显. T8样品 $(\mathrm{TOC}=0.39 \%)$ 和 T9样品 $(\mathrm{TOC}=0.60 \%)$ 虽然有明确的矿物峰，但基 线抬升幅度明显降低. $\mathrm{T} 1$ 样品 $(\mathrm{TOC}=1.01 \%)$ 荧光背景 进一步降低, 有机质的 $\mathrm{D}$ 峰与 $\mathrm{G}$ 峰基本上不受矿物的 影响.

从上述结果可以看出, 4 个样品的 $\mathrm{D}$ 峰和 $\mathrm{G}$ 峰的相 对强度随TOC含量的增加而增大, 说明MOA中较高的 TOC含量提高了拉曼光谱的信噪比(Sauerer等, 2017). 对于 TOC含量 $\geq 0.60 \%$ 的本研究高成熟度页岩, MOA 中有机质的拉曼信号强度足以掩盖其矿物背景的 影响.

图5为 $\mathrm{T} 1$ 样品 $(\mathrm{TOC}=1.01 \%)$ 中固体沥青与 $\mathrm{MOA}$ 激 
光拉曼光谱图的对比. 可见，虽然MOA中只含有极少 量的有机质, 而固体沥青基本上是一种纯有机物, 但两 者的激光拉曼光谱图基本上相同. Schopf等(2005)对前 寒武系含化石的燧石的研究也表明，对于从同一地质 单元岩石中的化石和腐质有机质中获得的拉曼光谱基 本上相同，这些光谱又非常类似于从其对应地层岩石 中分离出的干酪根的拉曼光谱. 这说明激光拉曼技术 对高成熟度岩石中含碳物质的高度敏感性. 即使岩石 中含有很低含量的有机质 (本研究系列样品 $\mathrm{TOC}=0.1 \%$ ), 也可通过激光拉曼检测到有机质的特征 拉曼吸收峰(G峰和D峰).

页岩MOA中有机质的分布具有非均质性，包括有 机质的数量及其亚显微结构. 为了进一步揭示MOA中 有机质和矿物的非均质性对其激光拉曼光谱特性的影 响, 图6展示了 9个样品(T1 T9)MOA和固体沥青的拉 曼光谱参数随TOC含量的变化. 可见, TOC含量主要 影响MOA参数的稳定性, 尤其是对低TOC样品较为明 显. $\mathrm{T} 7(\mathrm{TOC}=0.10 \%)$ 与 $\mathrm{T} 8$ 样品 $(\mathrm{TOC}=0.39 \%)$ 中 $\mathrm{MOA}$ 的 参数变化范围较大，与 $\mathrm{TOC}$ 含量较高的 $\mathrm{T} 1 \sim \mathrm{T} 6$ 样品 $(\mathrm{TOC}=1.01 \sim 4.59 \%)$ 中 MOA和固体沥青相比，其 F W H M - D 与 F W H M - G 明显较大, 而样品 T 9 $(\mathrm{TOC}=0.60 \%)$ 中 MOA的参数更接近这些高 $\mathrm{TOC}$ 含量 的样品. 随着TOC含量的进一步增加 $(\geq 1.01 \%)$, MOA 的数据与其共生固体沥青的数据基本重叠.

这些结果表明, $\mathrm{T} 7$ 和 $\mathrm{T} 8$ 样品数据的较大波动应主 要归因于其MOA中TOC含量较低, 而没有明显的证据 是与其MOA的亚显微干酪根组成的非均质性相关. Schito等(2017)也曾研究混合型(III与 II)干酪根样品的
激光拉曼成熟度参数, 发现其基本上不受干酪根类型 变化的影响. 根据本研究结果, 当样品 $\mathrm{TOC} \geq 0.6 \%, 5$ 个 测试点并可获得有效的激光拉曼参数, 对于较低 $\mathrm{TOC}$ 样品, 有必要增加测点数量, 用以获得具代表性的 数据.

\section{2 不同成熟度页岩MOA的激光拉曼图谱与参数}

图7为不同成熟度页岩样品MOA激光拉曼图谱. 随着成熟度增加, $\mathrm{D}$ 峰变窄、强度增加, 并有由不对称 向相对对称演化的趋势. 样品 $\mathrm{M} 1 \sim \mathrm{M} 10\left(B R_{\mathrm{o}}=\right.$ 1.71 4.05\%), D峰宽阔，强度较 $\mathrm{G}$ 峰弱. 对于更高成熟 度的两个样品(M11, M12), D峰的肩峰基本消失, 其相 对强度已大于 $\mathrm{G}$ 峰. 与 $\mathrm{D}$ 峰相比, $\mathrm{G}$ 峰形态较为简单, 其 演化规律也与 D峰不同. 从样品 $\mathrm{M} 1\left(B R_{\mathrm{o}}=1.71 \%\right)$ 到样品 $\mathrm{M} 9\left(B R_{\mathrm{o}}=3.88 \%\right), \mathrm{G}$ 峰逐渐变窄, 但随着成熟度进一步 增加(M10 M12), G峰又有变宽的趋势. 该现象同样见 于成熟度很高的其他地质样品, 与 $\mathrm{D} 2$ 峰 $\left(1620 \mathrm{~cm}^{-1}\right.$ 左 右)的出现与演化相关(如Beyssac等，2002; Kribek等, 2007; Kwiecinska等, 2010). 当有机质遭受的地质温度 $>360^{\circ} \mathrm{C}$ (Buseck和Beyssac，2014)或演化到初始石墨化 阶段(Schopf等，2015), D2峰开始与 $G$ 峰分离, 并形成 独立的峰. 本研究样品的D2峰没有突显出来, 其与 $\mathrm{G}$ 峰 重叠, 显然是因为其成熟度相对较低.

$\mathrm{D}$ 峰与 $\mathrm{G}$ 峰的峰位在热演化过程中的变化也体现 出明确的规律性. 随成熟度的增加, D峰的拉曼位移先 减小, 然后再增加, 转折点出现在 $B R_{\mathrm{o}}$ 为 $3.5 \sim 3.8 \%$ (图7). 从样品 $\mathrm{M} 1$ 到 $\mathrm{M} 10\left(B R_{\mathrm{o}}\right.$ 范围: 1.71 4.05\%), $\mathrm{G}$ 峰的峰位 变化不明显, 介于1600 1610 $\mathrm{cm}^{-1}$, 但随着成熟度的进

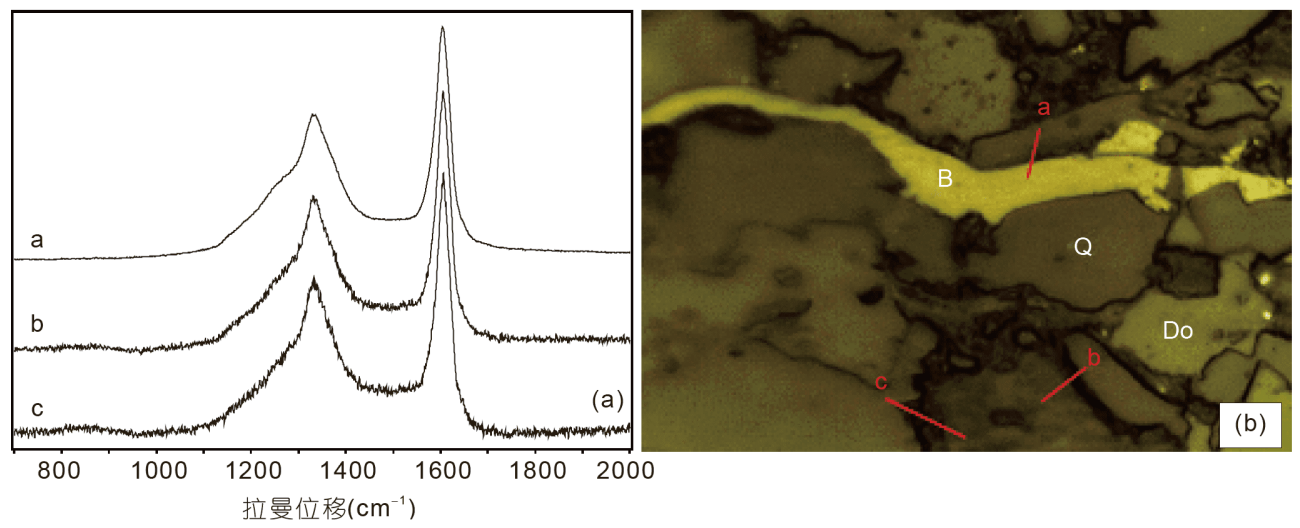

图 5 T1样品(TOC1.01\%)中固体沥青与MOA激光拉曼光谱图对比

(a) 三个测量点的拉曼谱图; (b) 50×空气物镜下测量样品的显微照片, 其中 B为沥青, $\mathrm{Q}$ 为石英, Do为白云石, a、 b 和 $\mathrm{c}$ 为三个测量点 

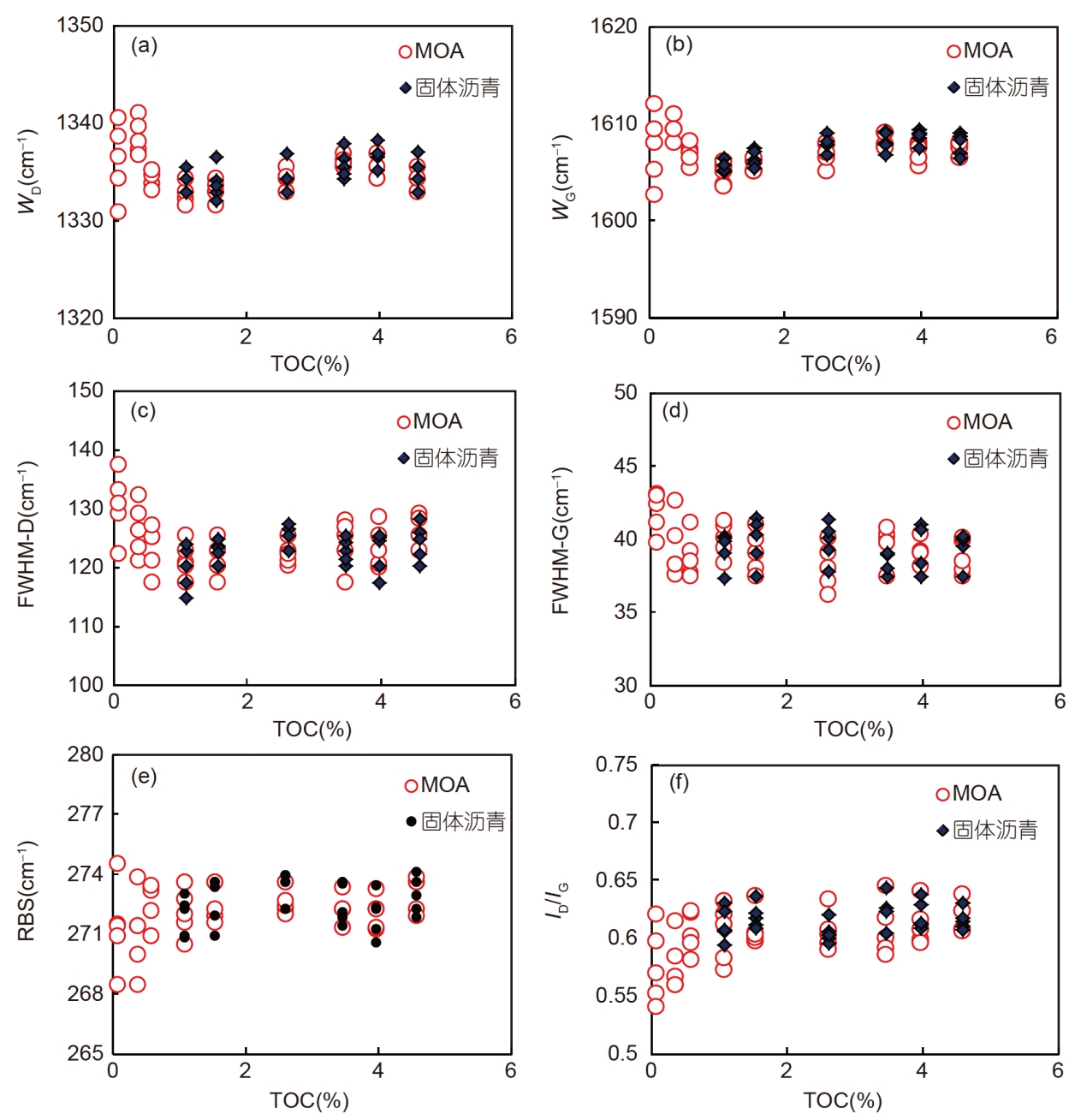

图 6 不同 TOC样品(T1 T9)MOA的激光拉曼参数随TOC的变化

一步增加(M11与M12样品), G峰的拉曼位移显著减小 (图7), 与 $\mathrm{G}$ 峰变宽相一致.

上述变化规律与成熟度的关系从相关参数中反映 得更加清楚(图8). 在本研究样品成熟度范围内, FWHM-D随着成熟度的增加而逐渐减少, 变化幅度为 $245 \sim 66 \mathrm{~cm}^{-1}$ (5点平均值, 以下相同). $B R_{\mathrm{o}}$ 从 $1.71 \%$ 增加 到 $3.88 \%, F W H M-G$ 从 $64 \mathrm{~cm}^{-1}$ 降低到 $39 \mathrm{~cm}^{-1} ; B R_{\mathrm{o}}$ 从 $3.88 \%$ 增加到 $4.57 \%, F W H M-G$ 又从 $39 \mathrm{~cm}^{-1}$ 增加到 $75 \mathrm{~cm}^{-1} . B R_{\mathrm{o}}$ 从 $1.71 \%$ 增加到 $3.73 \%, \mathrm{RBS}$ 从 $241 \mathrm{~cm}^{-1}$ 增 加到 $272 \mathrm{~cm}^{-1} ; B R_{\mathrm{o}}$ 从 $3.73 \%$ 增加到 $4.57 \%$, 其从 $272 \mathrm{~cm}^{-1}$ 减少到 $246 \mathrm{~cm}^{-1} . B R_{\mathrm{o}}$ 在1.71 3.58\%范围内, $I_{\mathrm{D}} / I_{\mathrm{G}}$ 与成熟 度的关系不明显, 变化范围为 $0.65 \sim 0.57$; 随着成熟度进 一步增加, $I_{\mathrm{D}} / I_{\mathrm{G}}$ 从 $0.57\left(B R_{\mathrm{o}}=3.58 \%\right)$ 快速增加到 $1.28\left(B R_{\mathrm{o}}\right.$ $=4.57 \%)$, 与 $B R_{0}$ 呈直线正相关.
页岩MOA上述激光拉曼参数的演化规律与王茂 林等(2015)报道的固体沥青完全具有可比性，与Liu等 (2013)报道的煤中镜质组也非常相似. 值得注意的是, 图8中的大多数参数在 $B R_{\mathrm{o}}$ 范围为 $3.5 \sim 3.8 \%$ 出现了拐点 或反转. 类似的现象也见于其他类型的有机质, 如煤 (Quirico等, 2005), 含干酪根的化石(Schopf等，2005), 页岩中镜质组(Lünsdorf, 2016). Henry等(2019b)根据文 献数据, 采用RBS、FWHM-G和其他拉曼参数详细的 论证了该拐点的存在, 并认为其出现在 $V R_{0}$ 约 $3.5 \%$, 对 应于地质温度约 $250^{\circ} \mathrm{C}$. Hou等(2019)认为有机质激光 拉曼光谱的这一“转折点”代表了其主要的化学体系与 结构的转变, 指示有机质的结构从非晶态碳转变为晶 态石墨, 并随着成熟度的进一步提高, 有机质的碳层 结构变得更加致密有序. 


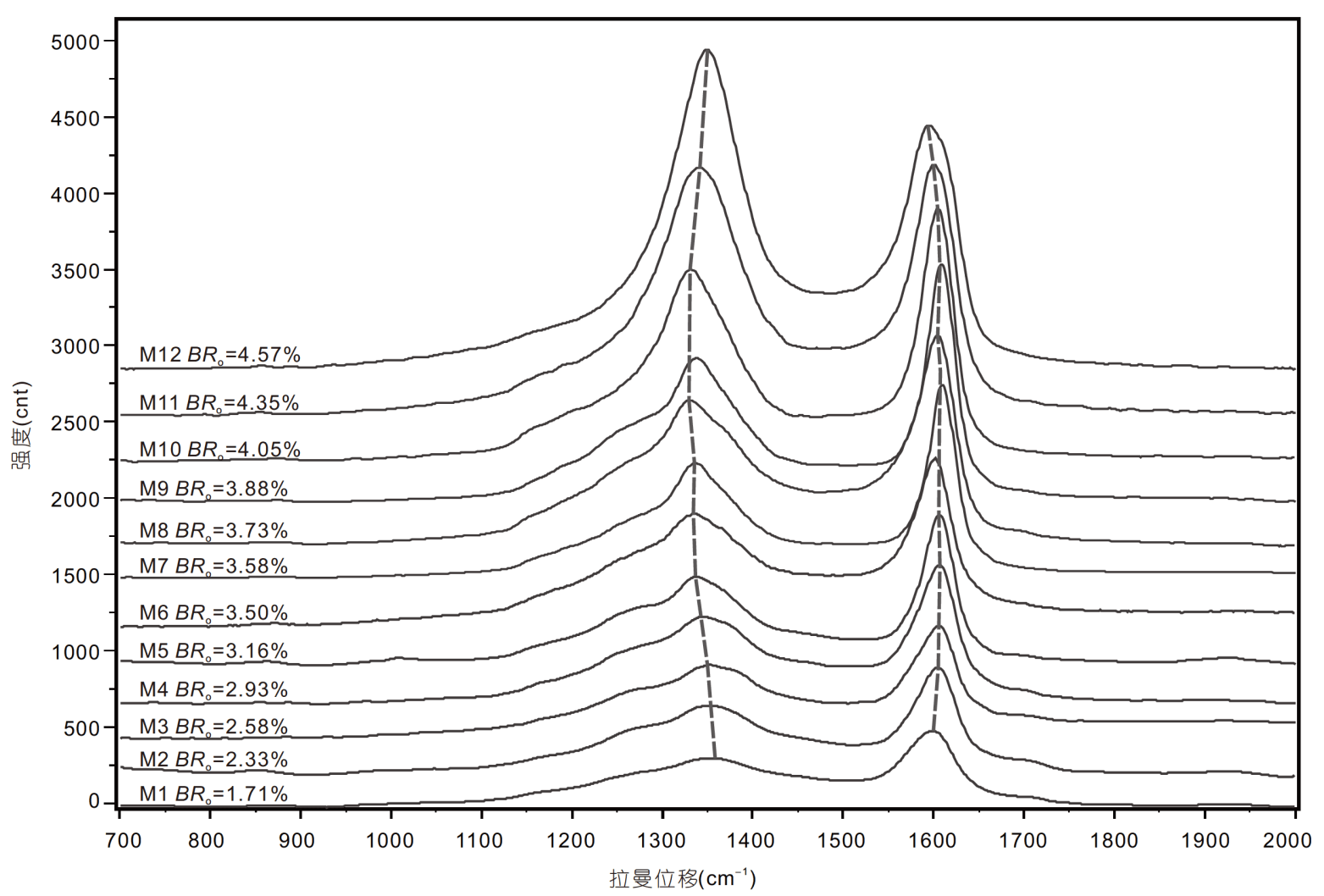

图 7 不同成熟度页岩样品MOA的激光拉曼图谱

所有谱图的强度尺度相同, 趋势线指示两个峰的位置随成熟度的变化

\section{3 页岩MOA激光拉曼成熟度参数}

根据上述讨论，可以认为页岩中MOA与固体沥青 一样, 其拉曼光谱参数可指示成熟度水平. 近年来, 文 献报道的激光拉曼成熟度参数包括: 峰位、峰位差、 半高宽、峰高比与峰面积比(Hinrichs等，2014; Zhou 等, 2014; Mumm和Inan, 2016; Chen等, 2017; Sauerer 等, 2017; Schito等, 2017; Schmidt等, 2017; Henry等, 2018). 用作MOA可能的成熟度指标, 本研究仅选择了 峰位、峰位差与峰高比, 而没有选择半高宽与峰面积 参数，这主要是因为前三项参数一般不会受到拟合方 法的影响, 对于相同的实验方法具有较好的可对比性.

根据图 8 的数据, 这些激光拉曼参数仅在一定成熟 度范围内与 $B R_{\mathrm{o}}$ 体现出较好的相关性： $W_{\mathrm{D}}$ 与 RBS对应 的 $B R_{\mathrm{o}}$ 范围为 $1.71 \sim 3.73 \%, I_{\mathrm{D}} / I_{\mathrm{G}}$ 对应的 $B R_{\mathrm{o}}$ 范围为 $3.58 \sim 4.5 \%$. 在上述 $B R_{\mathrm{o}}$ 范围内，采用直线回归方程拟 合了 $B R_{0}$ 与这些参数的相关性(图9). $W_{\mathrm{D}}$ 与 $B R_{\mathrm{o}}$ 呈线性 负相关，相关系数为 $0.92 . B R_{0}$ 与 $\mathrm{RBS} 、 I_{\mathrm{D}} / I_{\mathrm{G}}$ 均呈线性
正相关, 相关系数分别为 0.97 与 0.94 .

图10 反映的是 $B R_{\mathrm{o}}$ 与 $I_{\mathrm{D}} / I_{\mathrm{G}} 、 \mathrm{RBS} 、 W_{\mathrm{D}}$ 的关系. 可 见, 图8中所指示的成熟度演化的两个阶段在“转折点” 被清楚地分开，该“转折点”大致对应 $B R_{0}$ 值为 $3.73 \%$ 的 样品. 根据图10, 可以确定图9中适合于被测页岩样品 计算成熟度的相关公式.

本研究还对比了页岩MOA与固体沥青的激光拉 曼参数数据及其与 $B R_{0}$ 的相关关系(图9). 固体沥青的 数据来源于王茂林等(2015), 其中 11 个样品为本研究 的同一套样品，仅 $B R_{\mathrm{o}}=1.72 \%$ 的样品为从沉积岩中手 工挑选的纯固体沥青样品, 对应于本研究的M1样品 (表1). 这两套数据是在同一实验室、采用同一台仪器 在相同条件下测试，图谱拟合与参数的计算方法完全 相同. 因此，两套数据完全具可比性. 从图9中可见, MOA的回归线与固体沥青的回归线基本上重叠, 且具 有非常相近的相关系数, 这进一步表明MOA与固体沥 青激光拉曼光谱参数作为成熟度指标具有同等的 价值. 

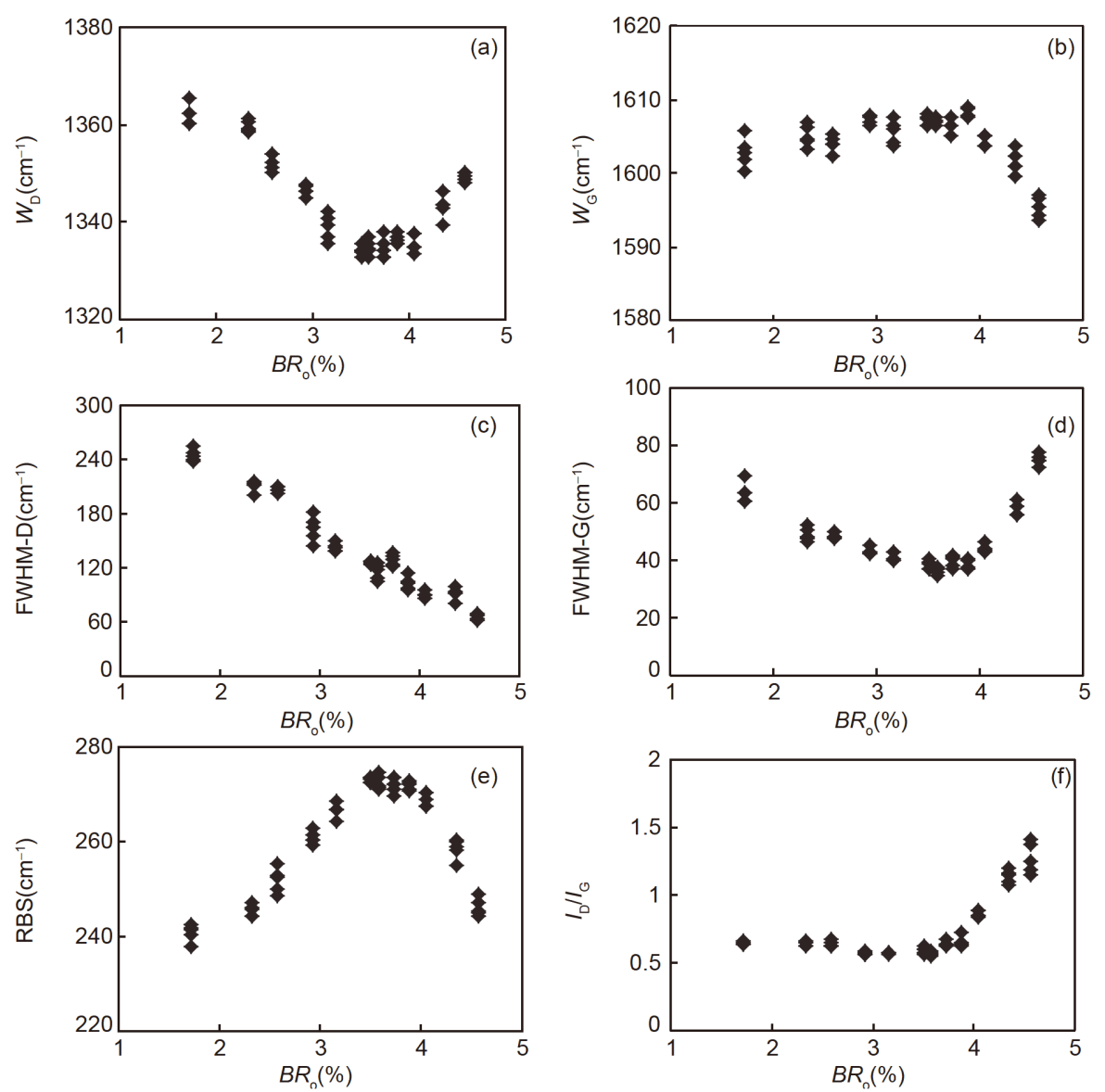

图 8 不同成熟度页岩样品MOA激光拉曼参数与固体沥青反射率 $\left(B R_{0}\right)$ 的关系

MOA中的有机质与固体沥青的成因不同. 对于本 研究页岩样品, MOA中的有机质为充分生成油气后的 残留干酪根，而固体沥青则是滞留油经热裂解形成的 固体残留物(Teichmüller，1986; Suárez-Ruiz等，2012). 根据本研究结果，当演化到高-过成熟度阶段后，两者 的激光拉曼图谱与参数非常相似. 关于这一点, 已有类 似的报道. 如Guedes等(2010)对煤中基质镜质组、粗 粒体及丝质体的研究表明，当 $V R_{0}>2 \sim 3 \%$ 后，三种显微 组分的激光拉曼参数逐渐趋于一致; 刘德汉等(2013) 对同一块下志留统页岩 $\left(B R_{\mathrm{o}}=3.2 \%\right)$ 中不同类型有机质 (类镜质组、固体沥青、微粒体)的研究也发现，他们 具有非常类似的激光拉曼图谱与参数. 结合不同显微 组分或不同类型干酪根演化到干气阶段后 $\left(V R_{0}\right.$ $>2.0 \%)$, 其光学性质和化学组成渐趋一致的规律 (Smith和Cook，1980; Tissot和Welte，1984), 有理由推 断，不同类型有机质的拉曼光谱特性随着成熟度的演
化规律可能与其光学性质及化学组成的演化规律相 似, 当演化到干气阶段后也会渐趋一致. 这也解释了为 什么本研究样品MOA与固体沥青具有非常相似的激 光拉曼光谱参数.

由于研究样品的限制，本研究没有对更低成熟度 的页岩样品 $\left(B R_{0}<1.71 \%\right)$ 的MOA进行测试. 根据Kelemen和Fang(2001)对两种类型干酪根( I 型与 II 型)人 工热模拟系列样品的激光拉曼研究, 当演化程度 $V R_{\mathrm{o}}$ $>1.5 \%$, 这两类干酪根的激光拉曼参数基本相同, 并与 天然演化的煤样相似. 这说明MOA的激光拉曼参数作 为成熟度指标可下延至 $V R_{0}$ 为 $1.5 \%$ 左右. 对于成熟度 更低的页岩样品, 其MOA的激光拉曼参数是否可用作 成熟度指标需要作进一步的研究.

\section{4 地质应用}

$\mathrm{MOA}$ 激光拉曼图谱参数应用于成熟度较高 $\left(B R_{\mathrm{o}}\right.$ 

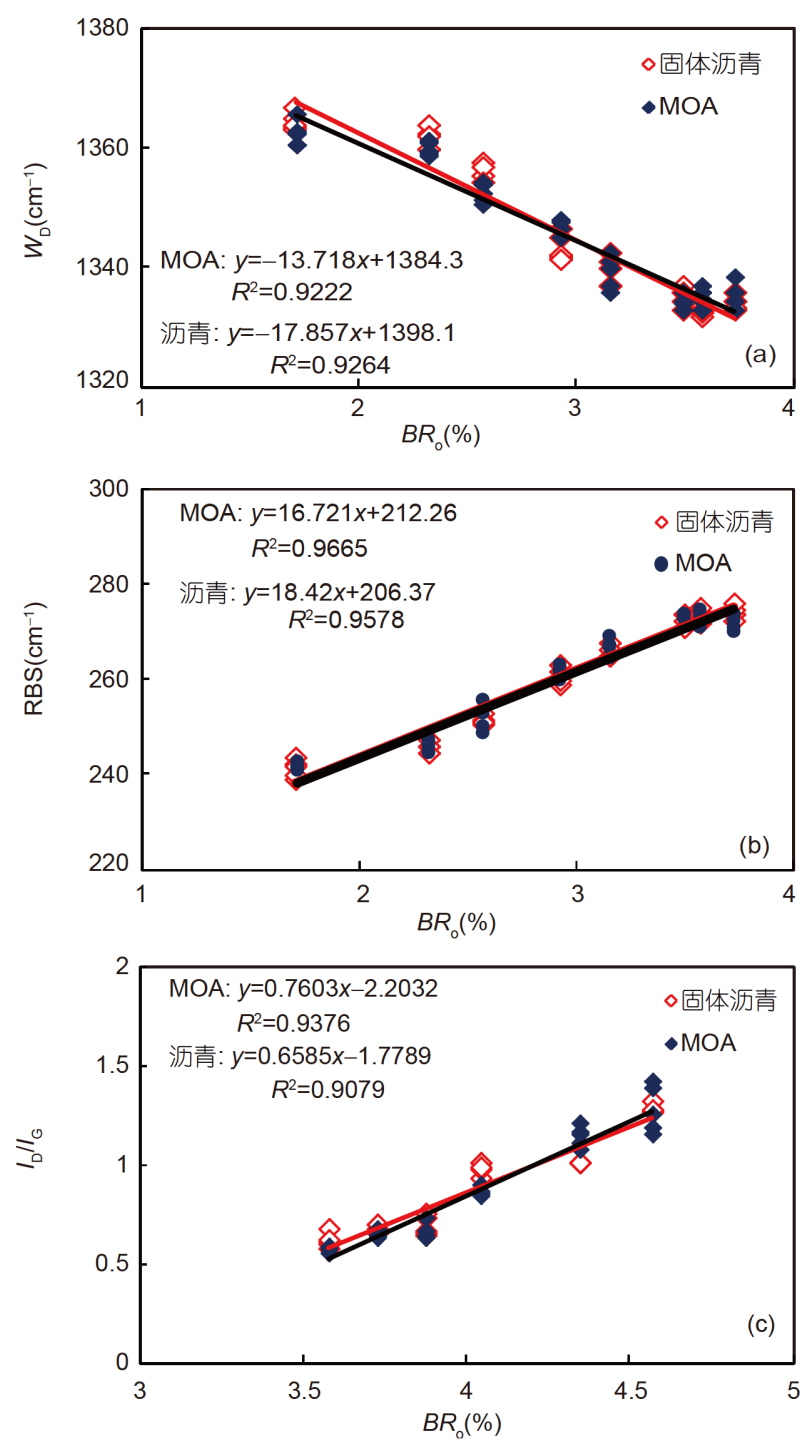

图 $9 M O A$ 和固体沥青的激光拉曼参数与 $B R_{0}$ 的关系 固体励青数据来源于王茂林等(2015)

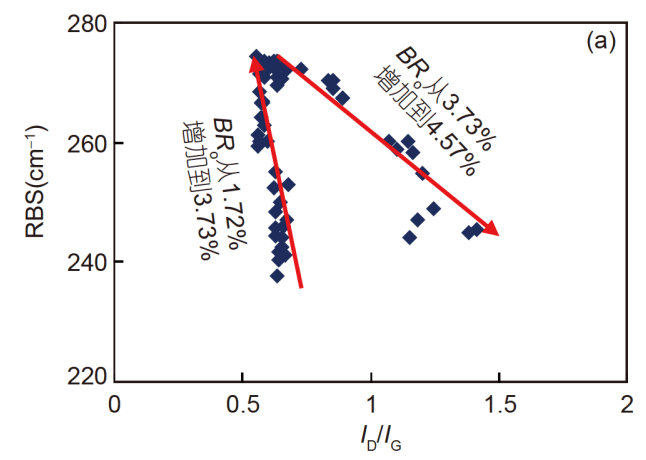

或 $\left.V R_{0}>1.5 \%\right)$ 页岩的成熟度评价将具有普遍的应用价 值. 尤其是某些下古生界与前寒武系海相页岩, 不仅成 熟度高, 而且缺乏可识别的显微组分(如类镜质组、固 体沥青、笔石体), MOA激光拉曼光谱参数可能是唯 一可应用的成熟度指标. 由于激光拉曼分析还可直接 采用全岩粉末样品(Quirico等, 2005; Guedes等, 2010), $\mathrm{MOA}$ 是页岩中的干酪根赋存的最主要形式, 因此基于 页岩粉末样品的激光拉曼分析(主要反映MOA)可使得 小数量样品及难制备块状光片或薄片样品(如岩屑)的 成熟度快速评价成为可能.

值得特别提及的是：虽然对煤和沉积岩中固体有 机质的激光拉曼光谱参数可指示其成熟度水平已经形 成了共识，但由于缺乏统一的方法(包括样品制备、测 试条件、图谱拟合与参数计算), 目前还处在自由探索 与研究阶段. 有必要借鉴建立镜质组反射率测定方法 国际标准化的经验, 在建立固体沥青激光拉曼光谱测 定的标准方法的基础上，实现该技术在页岩成熟度评 价的实际应用.

\section{4 结论}

本研究通过对取自中国南方高-过成熟海相页岩 $\left(B R_{\mathrm{o}}=1.71 \sim 4.57 \%\right)$ 中 $\mathrm{MOA}$ 激光拉曼图谱特征及参数的 研究, 主要获得如下认识与结论.

(1) 激光拉曼光谱可检测到页岩MOA中的微量有 机质(样品 $\mathrm{TOC}$ 可低至 $0.1 \%$ )反映的特征吸收峰 $(\mathrm{D}$ 与 $\mathrm{G}$ 峰), 且随着TOC的增加, $D$ 与 $G$ 峰的强度增加, 矿物峰 及矿物背景的强度减弱, 当TOC达到 $0.60 \%$, 可获得完 美的MOA拉曼图谱.

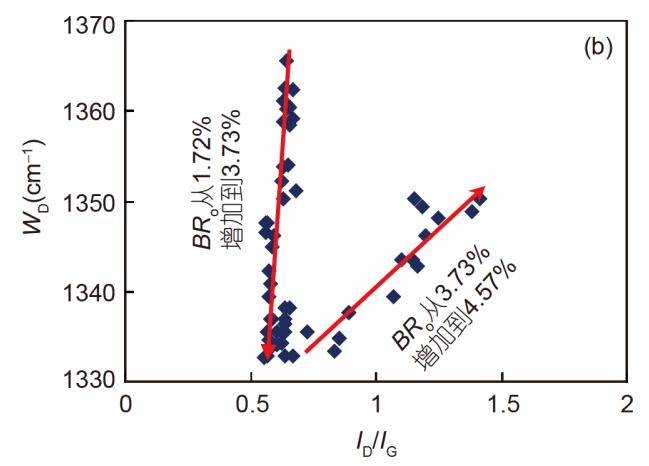

图 10 页岩样品MOA的拉曼参数 $I_{\mathrm{D}} / I_{\mathrm{G}}$ 与 $\mathrm{RBS}$ 及 $W_{\mathrm{D}}$ 的关系 
(2) 页岩中MOA与固体沥青的拉曼光谱参数具有 很好的可比性. 在一定的成熟度范围内, $B R_{\mathrm{o}}$ 与 $W_{\mathrm{D}}$ $\operatorname{RBS}\left(B R_{\mathrm{o}}<3.73 \%\right.$ ，可能可低至 $\left.1.5 \%\right) 、 I_{\mathrm{D}} / I_{\mathrm{G}}\left(B R_{\mathrm{o}}\right.$ $>3.58 \%)$ 均具有良好的线性相关性，相关系数达到 $0.92 \sim 0.97$.

(3) 页岩MOA的激光拉曼光谱参数为成熟度评价 提供了新的思路，尤其对于缺乏固体沥青的下古生界 或者前寒武系海相页岩的成熟度评价具有实际应用 价值.

致谢 感谢闵育顺研究员完成了部分样品的分析测试以 及两位评审专家对论文修改提出的宝贵意见.

\section{参考文献}

董大忠, 高世葵, 黄金亮, 管全中, 王淑芳, 王玉满. 2014. 论四川盆地 页岩气资源勘探开发前景. 天然气工业, 34: 1-15

柳晓飞, 尤静林, 王媛媛, Lu L, 解迎芳, 余立旺, 伏清. 2014. 澳大利 亚烟煤热解的拉曼光谱研究, 燃料化学学报, 42: 270-276

刘德汉, 肖贤明, 田辉, 闵育顺, 周秦, 程鹏, 申家贵. 2013. 固体有机 质拉曼光谱参数计算样品热演化程度的方法与地质应用. 科学 通报, 58: 1228-1241

刘树根, 邓宾, 钟勇, 再波, 雍自权, 孙玮, 杨迪, 姜否, 叶玥豪. 2016. 四川盆地及周缘下古生界页岩气深埋藏-强改造独特地质作用。 地学前缘, 23: 11-28

王茂林, 肖贤明, 魏强, 周秦. 2015. 页岩中固体沥青拉曼光谱参数作 为成熟度指标的意义. 天然气地球科学, 26: 1712-1718

王民, Li Z. 2016. 激光拉曼技术评价沉积有机质热成熟度. 石油学 报, 37: 1129-1136

杨斌, 贺晓苏, 徐云俊, 武金云, 杨兰英, 徐美茹. 1996. 中国南方下寒 武统烃源岩评价与油气资源力海相油气地质, 1: 31-38

张林, 魏国齐, 韩龙, 王立龙, 王东良. 2008. 四川盆地震旦系—下 古生界高过成熟度烃源岩评价. 石油实验地质, 30: 286-291

邹才能, 董大忠, 王社教, 李建忠, 李新景, 王玉满, 李登华, 程克明. 2010. 中国页岩气形成机理、地质特征及资源潜力. 石油勘探与 开发, 37: 641-653

Aoya M, Kouketsu Y, Endo S, Shimizu H, Mizukami T, Nakamura D, Wallis S. 2010. Extending the applicability of the Raman carbonaceous-material geothermometer using data from contact metamorphic rocks. J Metamorph Geol, 28: 895-914

Bernard S, Beyssac O, Benzerara K, Findling N, Tzvetkov G, Brown Jr. G E. 2010. XANES, Raman and XRD study of anthracene-based cokes and saccharose-based chars submitted to high-temperature pyrolysis. Carbon, 48: 2506-2516

Bertrand R, Malo M. 2001. Source rock analysis, thermal maturation and hydrocarbon generation in Siluro-Devonian rocks of the Gaspe Belt basin, Canada. Bull Can Pet Geol, 49: 238-261

Beyssac O, Goffé B, Chopin C, Rouzaud J N. 2002. Raman spectra of carbonaceous material in metasediments: A new geothermometer. J Metamorph Geol, 20: 859-871

Beyssac O, Goffé B, Petitet J P, Froigneux E, Moreau M, Rouzaud J N. 2003. On the characterization of disordered and heterogeneous carbonaceous materials by Raman spectroscopy. Spectroc Acta Pt A-Molec Biomolec Spectr, 59: 2267-2276

Buseck P R, Beyssac O. 2014. From organic matter to graphite: Graphitization. Elements, 10: 421-426

Bustin R M, Ross J V, Rouzaud J N. 1995. Mechanisms of graphite formation from kerogen: Experimental evidence. Int J Coal Geol, 28: $1-36$

Caricchi C, Corrado S, Di Paolo L, Aldega L, Grigo D. 2016. Thermal maturity of Silurian deposits in the Baltic Syneclise (on-shore Polish Baltic basin): Contribution to unconventional resources assessment. Ital J Geosci, 135: 383-393

Chalmers G R, Bustin R M, Power I M. 2012. Thermal maturity of Silurian deposits in the Baltic Syneclise (on-shore Polish Baltic basin): Contribution to unconventional resources assessment. AAPG Bull, 96: 1099-1119

Chen S, Wu D, Liu G, Sun R. 2017. Raman spectral characteristics of magmatic-contact metamorphic coals from Huainan Coalfield, China. Spectroc Acta Pt A-Molec Biomolec Spectr, 171: 31-39

Court R W, Sephton M A, Parnell J, Gilmour I. 2007. Raman spectroscopy of irradiated organic matter. Geochim Cosmochim Acta, 71: 2547-2568

Curtis J B. 2002. Fractured shale gas systems. AAPG Bull, 86: 19211938

Cuesta A, Dhamelincourt P, Laureyns J, Martínez-Alonso A, Tascón J M D. 1994. Raman microprobe studies on carbon materials. Carbon, 32: $1523-1532$

Endo S, Wallis S R, Tsuboi M, Torres De León R, Solari L A. 2012. Metamorphic evolution of lawsonite eclogites from the southern Motagua fault zone, Guatemala: Insights from phase equilibria and Raman spectroscopy. J Metamorph Geol, 30: 143-164

Engdahl Y Q A, Zhu S, Vajda V, McLoughlin N. 2015. Ultrastructural heterogeneity of carbonaceous material in ancient cherts: Investigating biosignature origin and preservation. Astrobiology, 15: 825-842

Ellery A, Wynn-Williams D, Parnell J, Edwards H G M, Dickensheets D. 2004. The role of Raman spectroscopy as an astrobiological tool in the exploration of Mars. J Raman Spectrosc, 35: 441-457

Ferrari A C, Robertson J. 2000. Interpretation of Raman spectra of disordered and amorphous carbon. Phys Rev B, 61: 14095-14107

Ferralis N, Matys E D, Knoll A H, Hallmann C, Summons R E. 2016. 
Rapid, direct and non-destructive assessment of fossil organic matter via microRaman spectroscopy. Carbon, 108: 440-449

Green P D, Johnson C A, Thomas K M. 1983. Applications of laser Raman microprobe spectroscopy to the characterization of coals and cokes. Fuel, 62: 1013-1023

Guedes A, Valentim B, Prieto A C, Rodrigues S, Noronha F. 2010. Micro-Raman spectroscopy of collotelinite, fusinite and macrinite. Int J Coal Geol, 83: 415-422

Henry D G, Jarvis I, Gillmore G, Stephenson M, Emmings J F. 2018. Assessing low-maturity organic matter in shales using Raman spectroscopy: Effects of sample preparation and operating procedure. Int J Coal Geol, 191: 135-151

Henry D G, Jarvis I, Gillmore G, Stephenson M. 2019a. A rapid method for determining organic matter maturity using Raman spectroscopy: Application to Carboniferous organic-rich mudstones and coals. Int J Coal Geol, 203: 87-98

Henry D G, Jarvis I, Gillmore G, Stephenson M. 2019b. Raman spectroscopy as a tool to determine the thermal maturity of organic matter: Application to sedimentary, metamorphic and structural geology. Earth-Sci Rev, 198: 102936

Hinrichs R, Brown M T, Vasconcellos M A Z, Abrashev M V, Kalkreuth W. 2014. Simple procedure for an estimation of the coal rank using micro-Raman spectroscopy. Int J Coal Geol, 136: 52-58

Hou Y, Zhang K, Wang F, He S, Dong T, Wang C, Qin W, Xiao Y, Tang B, Yu R, Du X. 2019. Structural evolution of organic matter and implications for graphitization in over-mature marine shales, south China. Mar Pet Geol, 109: 304-316

Jarvie D M. 2012. Shale resource systems for oil and gas: Part 1shale-gas resource systems. AAPG Mem, 97: 69-87

Jarvie D M, Hill R J, Ruble T E, Pollastro R M. 2007. Unconventional shale-gas systems: The Mississippian Barnett Shale of north-central Texas as one model for thermogenic shale-gas assessment. AAPG Bull, 91: 475-499

Jehlicka J, Urban O, Pokorný J. 2003. Raman spectroscopy of carbon and solid bitumens in sedimentary and metamorphic rocks. Spectroc Acta Pt A-Molec Biomolec Spectr, 59: 2341-2352

Kribek B, Sýkorová I, Pašava J, Machovič V. 2007. Organic geochemistry and petrology of barren and Mo-Ni-PGE mineralized marine black shales of the Lower Cambrian Niutitang Formation (South China). Int J Coal Geol, 72: 240-256

Kelemen S R, Fang H L. 2001. Maturity trends in Raman spectra from kerogen and coal. Energy Fuels, 15: 653-658

Kouketsu Y, Mizukami T, Mori H, Endo S, Aoya M, Hara H, Nakamura D, Wallis S. 2014. A new approach to develop the Raman carbonaceous material geothermometer for low-grade metamorphism using peak width. Island Arc, 23: 33-50
Kwiecinska B, Suárez-Ruiz I, Paluszkiewicz C, Rodriques S. 2010. Raman spectroscopy of selected carbonaceous samples. Int J Coal Geol, 84: 206-212

Li X, Hayashi J, Li C Z. 2006. FT-Raman spectroscopic study of the evolution of char structure during the pyrolysis of a Victorian brown coal. Fuel, 85: 1700-1707

Liu D H, Xiao X M, Tian H, Min Y S, Zhou Q, Cheng P, Shen J G. 2013. Sample maturation calculated using Raman spectroscopic parameters for solid organics: Methodology and geological applications. Chin Sci Bull, 58: 1285-1298

Liu X, You J, Wang Y, Lu L, Xie Y, Yu I, Fu Q. 2014. Raman spectroscopic study on the pyrolysis of Australian bituminous coal. J Fuel Chem Tech, 42: 270-276

Lünsdorf N K. 2016. Raman spectroscopy of dispersed vitriniteMethodical aspects and correlation with reflectance. Int J Coal Geol, 153: 75-86

Luo Q, Hao J, Skovsted C B, Luo P, Khan I, Wu J, Zhong N. 2017. The organic petrology of graptolites and maturity assessment of the Wufeng-longmaxi Formations from Chongqing, China: Insights from reflectance cross-plot analysis. Int J Coal Geol, 183: 161-173

Luo Q, Fariborz G, Zhong N, Wang Y, Qiu N, Skovsted C B, Suchý V, Hemmingsen Schovsbo N, Morga R, Xu Y, Hao J, Liu A, Wu J, Cao W, Min X, Wu J. 2019. Graptolites as fossil geo-thermometers and source material of hydrocarbons: An overview of four decades of progress. Earth-Sci Rev, 200: 103000

Lupoi J S, Fritz L P, Parris T M, Hackley P C, Solotky L, Eble C F, Schlaegle S. 2017. Assessment of thermal maturity trends in Devonian-Mississippian source rocks using Raman spectroscopy: Limitations of peak-fitting method. Front Energy Res, 5: 24

Marques M, Suárez-Ruiz I, Flores D, Guedes A, Rodrigues S. 2009. Correlation between optical, chemical and micro-structural parameters of high-rank coals and graphite. Int J Coal Geol, 77: 377-382

Mumm A S, Inan S. 2016. Microscale organic maturity determination of graptolites using Raman spectroscopy. Int J Coal Geol, 162: 96107

Petersen H I, Schovsbo N H, Nielsen A T. 2013. Reflectance measurements of zooclasts and solid bitumen in Lower Paleozoic shales, southern Scandinavia: Correlation to vitrinite reflectance. Int J Coal Geol, 114: 1-18

Popp J, Schmitt M. 2004. Raman spectroscopy breaking terrestrial barriers! J Raman Spectrosc, 35: 429-432

Quirico E, Rouzaud J N, Bonal L, Montagnac G. 2005. Maturation grade of coals as revealed by Raman spectroscopy: Progress and problems. Spectroc Acta Pt A-Molec Biomolec Spectr, 61: 23682377

Rahl J M, Anderson K M, Brandon M T, Fassoulas C. 2005. Raman 
spectroscopic carbonaceous material thermometry of low-grade metamorphic rocks: Calibration and application to tectonic exhumation in Crete, Greece. Earth Planet Sci Lett, 240: 339-354

Sadezky A, Muckenhuber H, Grothe H, Niessner R, Pöschl U. 2005. Raman microspectroscopy of soot and related carbonaceous materials: Spectral analysis and structural information. Carbon, 43: $1731-1742$

Sanei H, Haeri-Ardakani O, Wood J M, Curtis M E. 2015. Effects of nanoporosity and surface imperfections on solid bitumen reflectance (BRo) measurements in unconventional reservoirs. Int J Coal Geol, 138: 95-102

Sauerer B, Craddock P R, AlJohani M D, Alsamadony K L, Abdallah W. 2017. Fast and accurate shale maturity determination by Raman spectroscopy measurement with minimal sample preparation. Int J Coal Geol, 173: 150-157

Schoenherr J, Littke R, Urai J L, Kukla P A, Rawahi Z. 2007. Polyphase thermal evolution in the Infra-Cambrian Ara Group (South Oman Salt Basin) as deduced by maturity of solid reservoir bitumen. Org Geochem, 38: 1293-1318

Schito A, Romano C, Corrado S, Grigo D, Poe B. 2017. Diagenetic thermal evolution of organic matter by Raman spectroscopy. Org Geochem, 106: 57-67

Schmidt J S, Hinrichs R, Araujo C V. 2017. Maturity estimation of phytoclasts in strew mounts by micro-Raman spectroscopy. Int $\mathrm{J}$ Coal Geol, 173: 1-8

Schopf J W, Kudryavtsev A B, Agresti D G, Czaja A D, Wdowiak T J. 2005. Raman imagery: A new approach to assess the geochemical maturity and biogenicity of permineralized Precambrian fossils. Astrobiology, 5: 333-371

Schopf J W, Kudryavtsev A B. 2009. Confocal laser scanning microscopy and Raman imagery of ancient microscopic fossils. Precambrian Res, 173: 39-49

Smith G C, Cook A C. 1980. Coalification paths of exinite, vitrinite and inertite. Fuel, 59: 641-646

Sonibare O O, Haeger T, Foley S F. 2010. Structural characterization of Nigerian coals by X-ray diffraction, Raman and FTIR spectroscopy.
Energy, 35: 5347-5353

Suárez-Ruiz I, Flores D, Filho J C M, Hackley P C. 2012. Review and update of the applications of organic petrology: Part 1, geological applications. Int J Coal Geol, 99: 54-112

Teichmüller M. 1986. Organic petrology of source rocks, history and state of the art. Org Geochem, 10: 581-599

Tissot B, Welte D H. 1984. Petroleum Formation and Occurrence. 2nd ed. Heidelberg: Springer-Verlag. 669

Valentine B J, Hackley P C, Enomoto C B, Bove A M, Dulong F T, Lohr C D, Scott K R. 2014. Reprint of "Organic petrology of the Aptian-age section in the downdip Mississippi Interior Salt Basin, Mississippi, USA: Observations and preliminary implications for thermal maturation history". Int J Coal Geol, 136: 38-51

Wilkins R W T, Boudou R, Sherwood N, Xiao X. 2014. Thermal maturity evaluation from inertinites by Raman spectroscopy: The 'RaMM' technique. Int J Coal Geol, 128-129: 143-152

Wilkins R W T, Wang M, Gan H, Li Z. 2015. A RaMM study of thermal maturity of dispersed organic matter in marine source rocks. Int J Coal Geol, 150-151: 252-264

Xiao X M, Lin Z F, Sheng J Q. 1998. Characterization and origin types of immature and low-mature amorphous kerogen in terrestrial source rocks. Chin Sci Bull, 43: 241-244

Xiao X M, Wang F, Wilkins R W T, Song Z G. 2007. Origin and gas potential of pyrobitumen in the Upper Proterozoic strata from the Middle Paleo-Uplift of the Sichuan Basin, China. Int J Coal Geol, 70: $264-276$

Xiao X M, Wei Q, Gai H F, Li T F, Wang M L, Pan L, Chen J, Tian H. 2015. Main controlling factors and enrichment area evaluation of shale gas of the Lower Paleozoic marine strata in south China. Pet Sci, 12: 573-586

Zeng Y S, Wu C D. 2007. Raman and infrared spectroscopic study of kerogen treated at elevated temperatures and pressures. Fuel, 86: 1192-1200

Zhou Q, Xiao X M, Pan L, Tian H. 2014. The relationship between micro-Raman spectral parameters and reflectance of solid bitumen. Int J Coal Geol, 121: 19-25

(责任编委: 谢树成) 